Chapter 7

\title{
Generation of Induced Pluripotent Stem Cells from Dental Pulp Somatic Cells
}

\author{
Nelson F. Lizier, Irina Kerkis and \\ Cristiane V. Wenceslau \\ Additional information is available at the end of the chapter \\ http://dx.doi.org/10.5772/55856
}

\section{Introduction}

During early development, human dental pulp is originated from neural crest, which is a transient embryonic structure (Fig. 1). According to current knowledge, neural crest stem cells (NCSCs) have the capacity to self-renewal and display a developmental potential almost the same as embryonic stem (ES) cells (Kerkis and Caplan, 2012). These postmigratory NCSCs generate all craniofacial bones, the majority of the peripheral nervous system cells and tissues, as well as several non-neural cell types, such as smooth muscle cells of the cardiovascular system, pigment cells in the skin, cartilage, connective tissue, corneal epithelium and dental pulp among them. Although postmigratory, postnatal NCSCs are of restricted developmental potential they maintain functional characteristics resembling their embryonic counterparts and an ability to differentiate into a broad spectrum of cell types (Le Douarin et al., 2004, 2007, 2008; Dupin et al., 2007; Le Douarin \& Dupin, 2003, 2012).

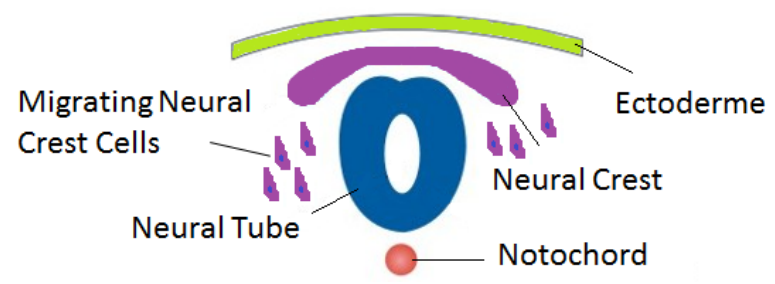

Figure 1. Early development of NCSCs. According to current knowledge, migrating neural crest cells are stem cells that display almost the same potential as ES cells. 
The marathon of induced pluripotent stem cells (iPSC) started when Yamanaka in 2006, by forcing the expression of certain pluripotent genes in fibroblasts, reversed them into a pluripotent state similar to ES cells (Takahashi \&Yamanaka, 2006). The main goal of iPSC generation is to create patient-specific cells, which would be advantageous for cell therapy due to immune compatibility (Ohnuki et al., 2009). Research involving the production of iPSC is being developed around the world. Production of iPSC opens new avenues for understanding human genetic diseases; embryogenesis and will likely have a great impact in drug screening and toxicological tests. However, fibroblasts, which were firstly used for iPSC production, present low efficiency and slow process of reprogramming. Moreover, these cells throughout all life are exposed to environmental factors, which can compromise their use as genetic models (Liu, 2008; McDevitt \& Palecek, 2008; Nishikawa et al., 2008; Yu \& Thomson; 2008; Zhao \& Daley, 2008; Maherali \& Hochedlinger, 2008; Ooi et al., 2012). Indeed, more immature somatic cells such as, postmigratory NCSCs, and adult stem cells isolated from young organism showed high efficiency of reprogramming (Zouboulis et al., 2008; Muchkaeva et al., 2012). Because of the possibility to isolate NCSCs from easily accessible tissue (e.g. baby teeth is discarded), the dental pulp derived somatic cells have become an ideal model system to study stem cell biology in diseases during different stages of the development (childhood, youth, middle-aged and old) with a special focus on non-invasive source of the cells for investigation of pediatric diseases (Kerkis \& Caplan, 2012; Lizier et al., 2012).

Our group isolated and fully characterized human immature dental pulp stem cells (hIDPSC), which is a very attractive cell type, from deciduous teeth (baby teeth) (Kerkis et al., 2006; Lizier et al., 2012). The hIDPSC present fibroblast-like morphology, retain characteristics of adult multipotent stem cells and express at least one of three transcription factors: Oct4, Nanog and Sox2 (Kerkis et al., 2006; Lizier et al., 2012). We also used these cells as an alternative source for iPSC derivation (Beltrão-Braga et al., 2011) (Fig. 2).Different research groups derived iPSC from dental pulp fibroblasts and stem cells from young, middle aged and old patients. The difference was observed between the protocols and efficiency of iPSC generation in all these studies (Yan et al., 2010; Tamaoki et al., 2010; Oda et al., 2010; Beltrão-Braga et al., 2011). The present chapter is focused on comparative investigation of the methods and efficiency of iPSC generation from dental pulp stem cells and fibroblasts (control). Differentiation potential, assuredness and the future perspectives of the use of these iPSC derived from dental pulp stem cells in basic research and in biotechnology will also be broadly discussed.

\section{Dental pulp somatic cells used for iPSC generation}

Human dental tissues are rich in stem cells (Giordano et al., 2011; Kerkis \& Caplan, 2012). Different research groups isolated and characterized several types of stem cells used for iPSC generation: (i) from apical papilla (Yan et al., 2010), (ii) from dental pulp of primary exfoliated deciduous teeth (Yan et al., 2010; Beltrão-Braga et al., 2011) and (iii) from wisdom teeth (Tamaoki et al., 2010; Oda et al., 2010). SCAP (stem cells from apical papilla) were obtained from tissue at the apex of a tooth root (Yan et al., 2010). SHED (stem cells from human exfoliated deciduous) and IDPSC (immature dental pulp stem cells) were derived from exfoliated 
deciduous teeth (Miura et al., 2003; Kerkis et al., 2006). DPCs (dental pulp cells) and MStCs (mesenchymal/stromal cells) were isolated from human third molars by two independent groups (Takeda et al., 2008; Ikeda et al., 2008). All these cell types present fibroblast-like morphology and however differ in methods of isolation, show significant difference in expression pattern of stem cell markers and in purity of isolated population (Yan et al., 2010; Beltrão-Braga et al., 2011; Tamaoki et al., 2010; Oda et al., 2010).

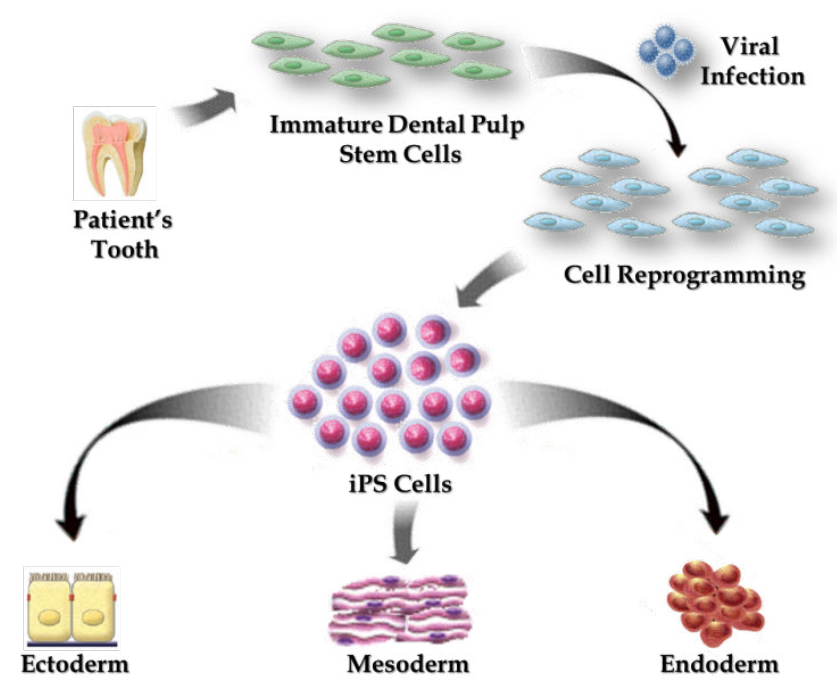

Figure 2. iPSC technology. The iPSC production can be induced by forcing the expression of certain pluripotent genes.

\subsection{Transduction and reprogramming}

For reprogramming of SHED/SCAP/DPSCs, heterogeneous primary human dental stem/ progenitor cell population at passages 2 and 3 were used (Yan et al., 2010). These populations were tested for their cell surface marker expression by flow cytometry and they were positive for STRO-1, CD146, CD73, CD90, CD105 and negative for CD14, CD34, and CD45, showing typical immunophenotype of mesenchymal stem cells (MSC) (Friedenstein et al., 1976; Caplan, 1991). The first study used four factor genes for reprogramming, such as c-Myc [Myc protooncogene protein], Klf4 [Krüppel-like factor], Oct4 [octamer-binding transcription factor 4], and Sox2 [(sex determining region Y)-box 2] into pLenti6.2/C-Lumio/V5-DEST vector system. Although the cells started to present morphological changes (fibroblastic to epithelial cell-like transition), the reprogramming process failed. Further, lentiviral vectors pSin-EF2-gene-Pur carrying 1 of the 4 factors Lin28 [Lin-28 homolog A], Nanog (Nanog homeobox), Oct4, and Sox 2 were used and first ES-like colonies were obtained. To improve reprogramming efficiency, human genes c-Myc, Klf4, Oct4, and Sox2 were subcloned into the vector pMXs and 
produced retrovirus was used for second round of transduction. Human fibroblasts, used as a control in this study, were not able to undergo reprogramming under proposed conditions.

Retroviruses expressing four Oct3/4, Sox2, Klf4, and c-Myc or three (without c-Myc) factors were used for reprogramming DPCs from wisdom teeth and from human dermal fibroblasts (HDFs), which was performed according to the methods previously described (Takahashi et al., 2007). Another group, which used MStCs from wisdom teeth, also demonstrated successful reprogramming of these cells with pMXs retrovirus vectors containing three human Oct3/4, Sox2, and Klf4 factor genes (Oda et al., 2010).

IDPSC is a homogeneous population in respect of the expression of MSCs (Friedenstein et al., 1976; Caplan, 1991) markers, such as CD73, CD105, nestin and vimentin. Within IDPSC population, several cells also express Oct3/4 and Nanog (Kerkis et al., 2006; Lizier et al., 2012). To reprogram IDPSC, our group used four Yamanaka's factors (Klf4, Oct4, c-Myc and Sox2) and previously established protocol (Takahashi et al., 2007; Beltrão-Braga et al., 2011).

\subsection{Formation of ES cell-like colonies and expansion}

There are several important points that should be considered when iPSC are isolated and expanded: (i) the use of mouse embryonic fibroblasts (MEF) as a feeder layer, (ii) the efficiency of reprogramming and (iii) the efficiency of expansion (Takahashi \& Yamanaka, 2006; Lewitzky \& Yamanaka, 2007; Bilic \& Belmonte, 2012). Isolation of iPSC on MEF limits the manipulation and further clinical application of these cells. Thus, isolation and expansion of iPSC without MEF is an important step, which avoids contamination of human cells with animal products. Efficiency of reprogramming depends on different factor, such as gene expression profile of cells, which were used in experiments. It has been shown that more immature cells undergo this process more efficiently, then committed or terminally differentiated cells (Zouboulis et al., 2008; Muchkaeva et al., 2012). And finally, during reprogramming, the cells receive different number of reprogramming factors and/or they did not respond equally to this process, therefore multiple ES-like did not complete reprogramming or non-ES cell-like colonies raised (Aasen et al., 2008; Marchetto et al., 2009). SHED/SCAP/DPSCs/DPCs/ MStCs-derived iPSC were obtained using MEF as a feeder layer (Yan et al., 2010; Tamaoki et al., 2010; Oda et al., 2010). IDPSC-derived iPSC were shown to be obtained under both conditions: feeder-free on matrigel-coated dishes and on MEF (Fig. 3) (Beltrão-Braga et al., 2011). It seems that time-course of reprogramming of different cell types varied in accordance with age of cell donor, cell type and number of factors used. Thus, SHED/SCAP/DPSCsderived iPSC, showed the formation of the first colonies $\sim 2-3$ weeks after gene transduction. DPCs-derived iPSC were reprogrammed in $\sim 14$ days, when 4 factors were used and in $\sim 20-25$ days, when reprogramming was performed with only 3 factors. MStCs-derived iPSC were reprogrammed in $\sim 25$ days, while IDPSC-derived iPSC demonstrated the formation of first colonies at $\sim$ day 5-11. All studies demonstrated that efficiency of iPSC derivation from dental pulp tissues is higher than that from human dermal fibroblast and primary gingival fibroblasts (Yan et al., 2010; Tamaoki et al., 2010; Oda et al., 2010; Beltrão-Braga et al., 2011). Because the MStCs were a heterogeneous cell population, Oda and co-authors (2010) additionally used clonally expanded MStCs in reprogramming experiments. They observed that reprogramming 
efficiency in clonally expanded MStCs was higher and it correlates with cell proliferative ability. The clones, which showed higher proliferative ability, demonstrated a rate of reprogramming 30-100-fold higher than HDFs and 7-fold higher than clones with lower proliferative ability. The IDPSC also present high reprogramming efficiency and no difference was observed between the cells from both donors. Therefore, difficulties in reprogramming SHED/ SCAP/DPSCs can be related with cell heterogeneity of original populations (Yan et al., 2010).
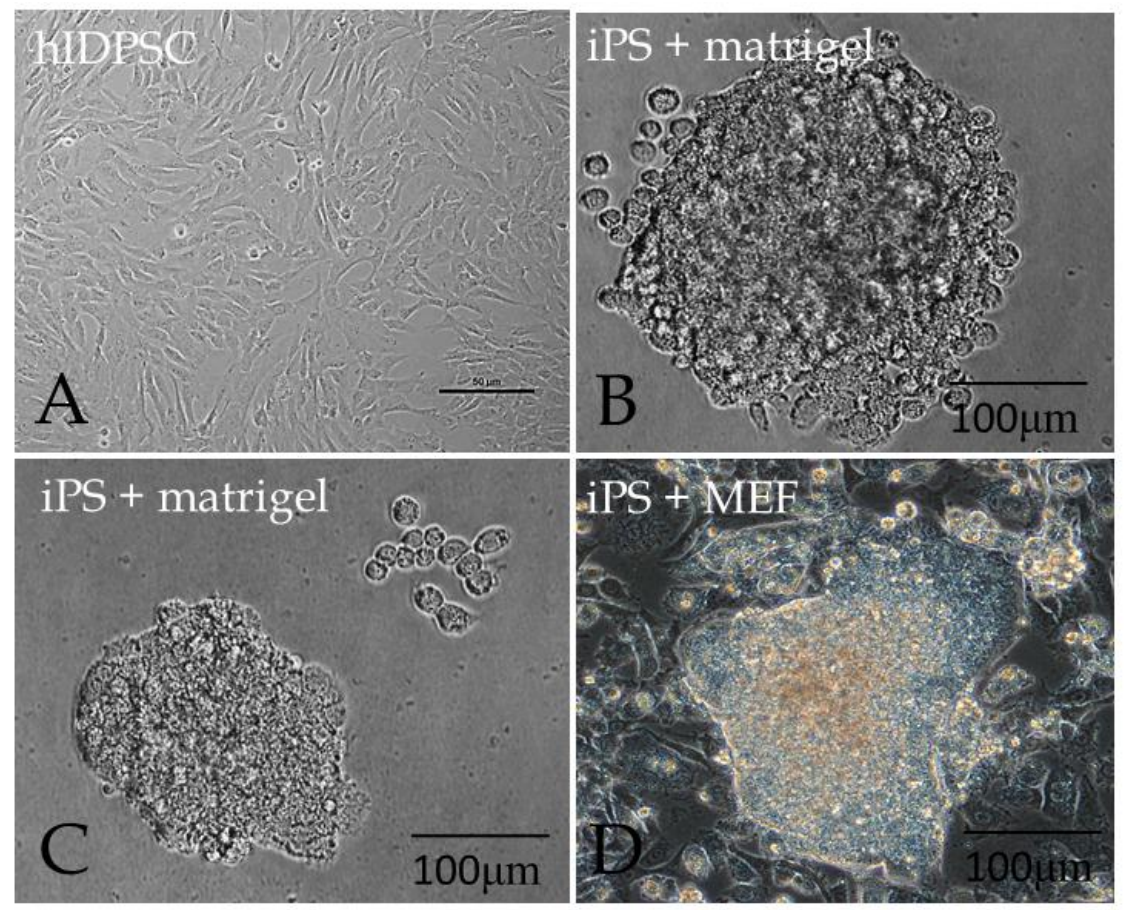

Figure 3. hIDPSC-derived iPSC. (A) Representative figure of morphological characteristics of hIDPSC in vitro culturing (light microscopy). (B-D) iPSC derivations were shown to be obtained under both conditions: feeder-free, on matrigelcoated dishes (B and C) and on MEF (D).

Furthermore, the aging process influences all organs, tissues and cells of organism. The studies showed that this factor is also important for cells reprogramming (Zouboulis et al., 2008; Banito et al., 2009). SHED/SCAP/DPSCs/DPCs/MStCs/IDPSC were isolated from young donors of variable ages $7,10,12,13,14$ 16, 19, 20 and 24 years old. The difference in efficiency of iPSC generation was observed between MStCs isolated from third molars of 10-, 13-, and 16-yearold donors. More efficient reprogramming was observed when MStCs from the 10-year-old donor were used. Similar observation was made by Tamaoki and co-wokers (2010). In our study we used IDPSC from 7 years old donors and we observed rapid and efficient reprogramming in both cell populations. 


\subsection{Provirus integration}

Viral vectors are commonly used to deliver genetic material into cells, which can be performed in vivo (living organism) or in vitro (cell culture). Delivery of genes by a virus is efficient, however with respect to safety, it is ideal not to use lentivirus and retrovirus vectors, since they can integrate into the host DNA. As opposed to lentiviruses and retroviruses, adenoviral DNA does not integrate into the genome and are considered to be safer (Tamaoki et al., 2010). All studies performed with stem cells of dental tissue origin used lentivirus and/or retrovirus vectors for reprogramming. Yan and co-workers (2010) examined the presence of transgenes in the genome of iPSC clones isolating genomic DNA and generating primers specific for each transgene. They showed that the 4 factors were all integrated into the genome of the transduced SHED/SCAP/DPSC-iPSC. Other study, which used MStCs from wisdom teeth for reprogramming, did not provide any records about viral vectors integration (Tamaoki et al., 2010), while another group demonstrated retroviral silencing (Oda et al., 2010). Beltrão-Braga and coauthors (2011), which used retroviral vector for reprogramming, showed the lack of transgene expression by RT-PCR analyses in iPSC-derived from IDPSCs.

\subsection{Characterization of SHED/SCAP/DPSCs/DPCs/MStCs/IDPSC-derived iPSC}

\subsubsection{Expression of pluripotent stem cell markers}

As expected in all studies, the iPSC obtained from tissues of dental origin, which showed ESlike cells morphology, express key markers of pluripotent stem cells in an appropriate manner. Immunofluorescence study demonstrates uniform expression of these antigens in iPSC colonies derived from different types of dental stem cells. Transcription factor proteins as Oct3/4, Nanog, Sox 2 demonstrate nuclear localization, while cell surface markers, such as stage specific embryonic antigen (SSEA) 3 and SSEA4, as well as cell surface antigens of human embryonic carcinoma cells (TRA-1-60 and TRA-1-81) show cell surface localization. Appropriate expression of transcription factors Klf4, c-Myc, Lin28, that were part of the transgene used for reprogramming, also was observed (Yan et al. 2010; Tamaoki et al., 2010; Oda et al., 2010; Beltrão-Braga et al., 2011) (Fig. 4).

\subsubsection{Expression of molecular markers of pluripotent stem cells}

Only one study performed quantitative PCR analysis before and after reprogramming for endogenous expression of Oct4, Nanog and Sox 2 genes and compared the expression level of all these genes with those in pluripotent human ES cells. Albeit we revealed a tendency for increasing of expression of pluripotent factors Oct4, Nanog and Sox2, when compared to nonreprogrammed cells (18\%, $1 \%$ and $2 \%$, respectively), it was significantly lower $20 \%$ (Oct 4$), 10 \%$ (Nanog) and 40\% (Sox2) in comparison with human ES cells (100\% - Oct4, Nanog, Sox 2$)$ (Beltrão-Braga et al., 2011). Other studies did not provide any data about expression of these key markers in SHED/SCAP/DPSCs/DPCs/MStCs - derived iPSC in comparison with ES cells (Yan et al., 2010; Tamaoki et al., 2010; Oda et al., 2010). However, Oda and colleagues (2010) demonstrated that expression levels of Oct4, Nanog, Sox2, Klf4, c-Myc, Lin28 and P53 was 
higher in iPSC derived from clonally isolated MStCs, when compared with parental cell lines, iPSC-derived from these lines and HDFs, used as a control.
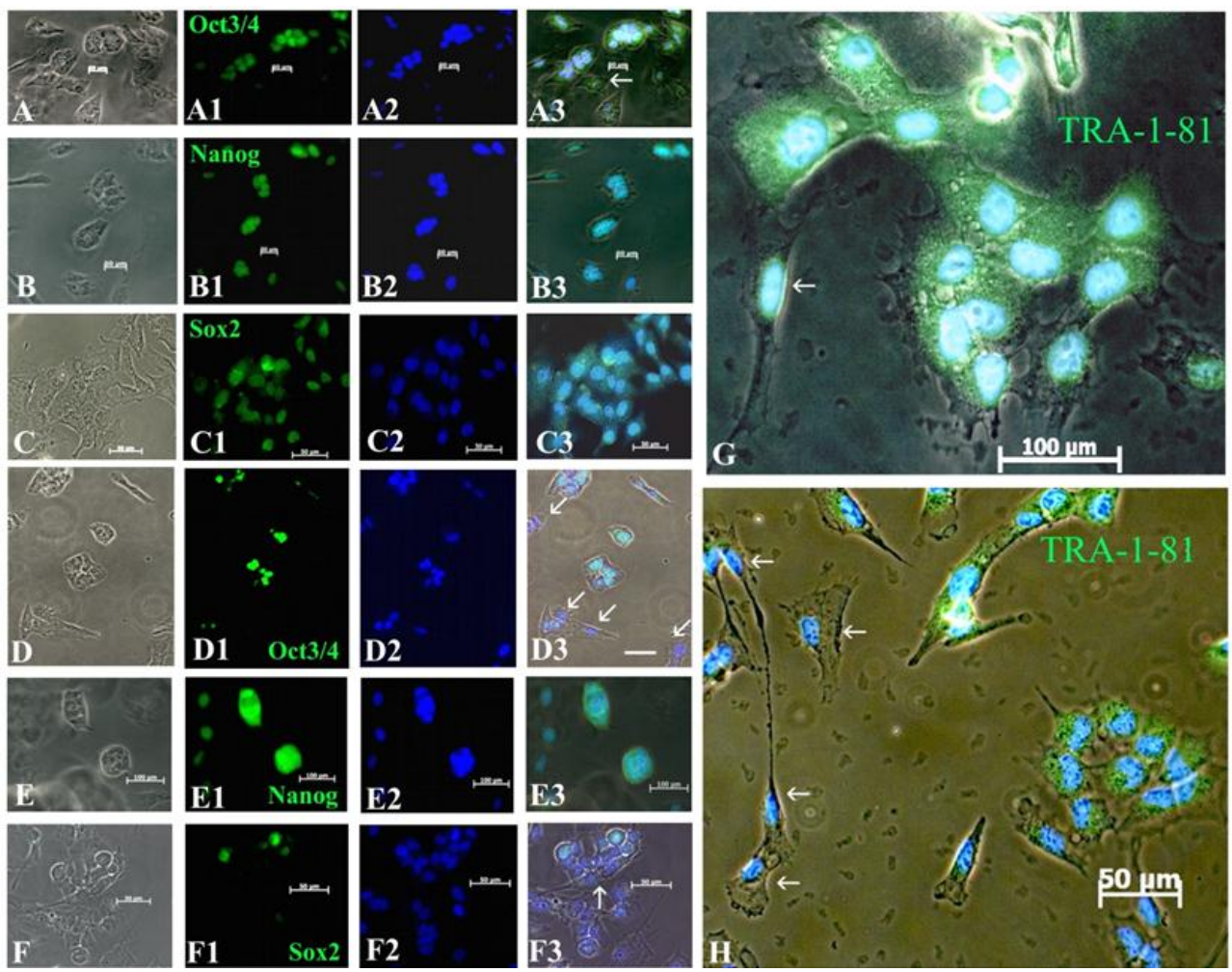

Figure 4. Expression of Oct3/4, Nanog, Sox2 and TRA-1-81 proteins in two lineages of hIDPS-IPSC five days after transduction with four factors. In A-C3,G) hIDPS-IPSC1 and D-F3,H) hIDPS-IPSC2 are presented, both showing multiple small colonies, which already express hallmarks of pluripotent cells, such as, A-A3) and D-D3) Oct3/4; B-B3) and E-E3) Nanog, C-C3) and F-F3) Sox2; G) and H) TRA-1-81, respectively. Nucleus stained with DAPI (blue). Note, that Oct3/4, Nanog, Sox2 present nuclear, while TRA-1-81 presents cytoplasm localization. Several cells, which did not present expression of these proteins and served as a control, are indicated by white arrows. Confocal Microscopy: A-F) Differential interference contrast (DIC); A1-F1 and A2-F2) Fluorescent microscopy (Fm); A3-F3, G, H) DIC+Fm. Scale Bars: A-D3, $\mathrm{F}-\mathrm{F} 3, \mathrm{H}=50 \mu \mathrm{m} ; \mathrm{E}-\mathrm{E} 3, \mathrm{G}=100 \mu \mathrm{m}$.

Yan et al., (2010) quantified by real-time PCR the expression levels of endogenous Klf4 and cMyc. Klf4 showed relative higher expression in DPC lines than in HDFs, however lower than in ES cells. Endogenous c-Myc expression in most DPC lines was also slightly higher than that in HDFs and in a few iPSC clones were close to ES cells. In contrast, Oda et al., (2010) observed low expression of Klf4 in high reprogramming cells, which was unexpected, once Klf4 is a reprogramming factor. Yan et al., (2010) showed that endogenous Klf4 expression level determined by real-time PCR did not completely correlate with the reprogramming efficiency of each DPCs (wisdom teeth) line. It is noteworthy that highly expression of KLF4 was 
previously reported in senescent cells and terminally differentiated cells (Shields et al., 1996; Conkright et al., 1999). Taken together, these data suggest that endogenous Klf4 expression may not be the single factor in charge for the reprogramming efficiency to MSCs derived from wisdom teeth.

\subsubsection{Searching for new factors of reprogramming}

Oda et al., (2010) tried to find the additional unknown factor(s) that could help in the cell reprogramming. They focused their study on practically two genes: PAXIP1 (or PTIP) and PARP. PAXIP1 acts as component of a histone H3 lysine four (H3K4) methyltransferase complex (Cho et al., 2007; Patel et al., 2007) and has a role in DNA double-strand break repair (van Attikum and Gasser, 2009). It was demonstrated that efficient reprogramming of pluripotent gene (Oct3/4, Sox2) expression is associated with H3K4 methylation in mouse somatic cell nuclei transplantation into amphibian oocytes (Murata et al., 2010). The expression of this gene was about $30 \%$ more in the high reprogramming cells than in low reprogramming as well as 3-4 times more in iPSC when compared with each parental cell line. PARP-1 belongs to PARP family being the most abundant member and is responsible for $>85 \%$ of nuclear PARP activity modifying histone structure through DNA-dependent "PARylation". Higher expression of PARP-1 was also seen after induction of reprogramming in cells derived from wisdom teeth. The authors supposed that due to possible conformational change of chromatin by direct/indirect actions of chromatin modification proteins such as PAXIP1 and possibly PARP-1, high iPSC generation clones may be accessible for reprogramming factors. However, further investigation is needed to illuminate the iPSC reprogramming mechanisms using these genes.

\subsubsection{Methylation status of cytosine guanine dinucleotides (Cp $G$ )}

The methylation status of $\mathrm{CpG}$ in the promoter regions of Nanog and Oct 4 was examined using bisulfite DNA sequencing method in two studies (Yan et al., 2010; Oda et al., 2010) and of Nanog in one study (Tamaoki et al., 2010). They showed that parental MStCs from wisdom teeth were highly (Oct3/4) or partially (Nanog) methylated and the iPSC-derived from these cells were highly unmethylated, suggesting that these promoters were active after cells reprogramming. In contrast, the analysis of iPSC clones derived from DPSCs (wisdom teeth) and SHED (deciduous teeth) showed that Nanog promoter had similar or slightly higher number of methylated sites, than their non-transduced counterparts. The SHED-/DPSC-iPSC had less methylated sites of Oct3/4 promoter than the non-transduced cells (Yan et al., 2010).

\subsubsection{Telomerase activity}

Telomerase activity is known to be highly activated in ES cells in order to maintain the integrity of chromosome structure. After reprogramming, SHED- (deciduous teeth) SCAP-, and DPSC (wisdom teeth) - iPSC showed telomerase activity very close to ES cells and a lot more in comparison to their non-transduced counterparts (Yan et al., 2010). Parental DPSCs (wisdom teeth) showed low telomerase activity whereas in each iPSC telomerase activity was high (Oda et al., 2010). 


\subsubsection{Karyotype analysis of dental stem cell-derived iPSC}

Karyotype study has been performed by all authors and demonstrated that karyotype of reprogrammed cells remained unchanged (Fig. 5). Overall, during reprogramming of stem cell from dental pulp, numerical and gross structural chromosomal abnormalities were not detected (Yan et al., 2010; Tamaoki et al., 2010; Oda et al., 2010; Beltrão-Braga et al., 2011).
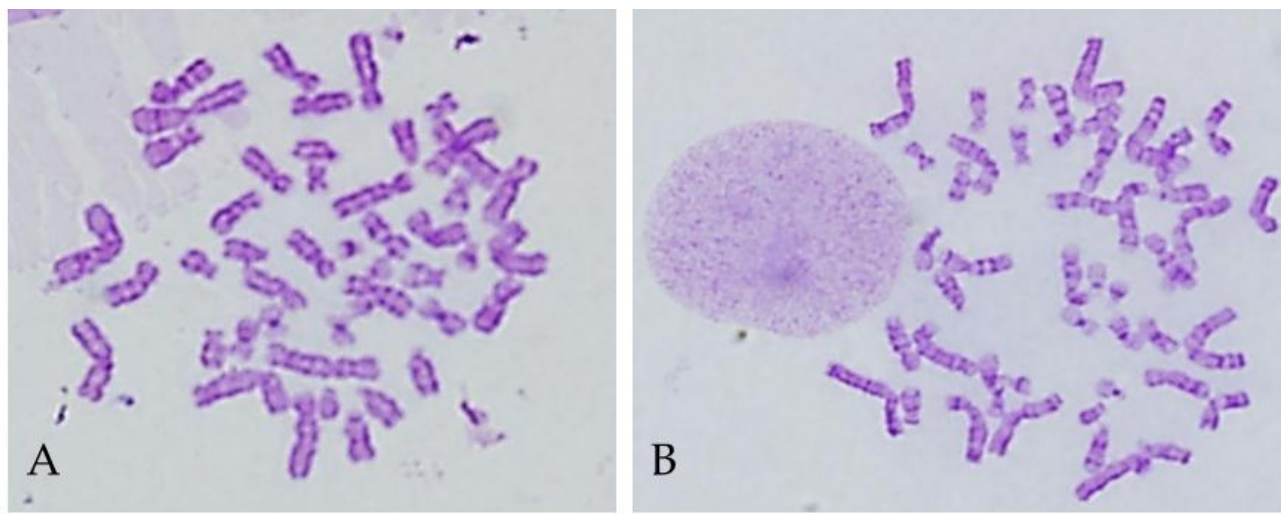

Figure 5. Representative figures of karyotype analysis of both hIDPSC and hIDPS-iPSC: Routine Giemsa staining did not reveal any numerical changes in chromosome number (A and B) and any chromosomal structural changes (B). Magnification 63X. Differential interference contrast (DIC)

\subsection{Differentiation of SHED/SCAP/DPSCs/DPCs/MStCs/IDPSC-iPSC}

\subsubsection{Embryoid body formation and in vitro differentiation}

Similar to human ES cells, iPSC require the formation of embryoid bodies (EB) in order to undergo in vitro differentiation into various cell types. All studies confirm successful differentiation of iPSC-derived from different types of dental stem cells into all three germ layers (Yan et al., 2010; Tamaoki et al., 2010; Oda et al., 2010; Beltrão-Braga et al., 2011). As expected, the majority of iPSC formed EBs with cystic cavities. The histological analysis demonstrates that EBs differentiated into different cell types of ectodermal, mesodermal, or endodermal origin, which was demonstrated using antibodies against specific proteins, which is expressed in each of three germ layers. It has been shown that after reprogramming, cells tend to maintain their original commitment. Dental pulp stem cells are multipotent stem cells derived from neural crest and they showed strong commitment into neural lineages. Therefore, neural differentiation is widely presented in all iPSCderived from different types of dental stem cells. Under appropriate neurogenic culture medium, the EBs developed into the cells with neural-like morphology (Fig. 6), which express such markers as nestin, $\beta$-tubulin III (TUJ1), neuron-specific Enolase (NSE) and glial fibrillary acidic protein (GFAP) (Fig. 6). 

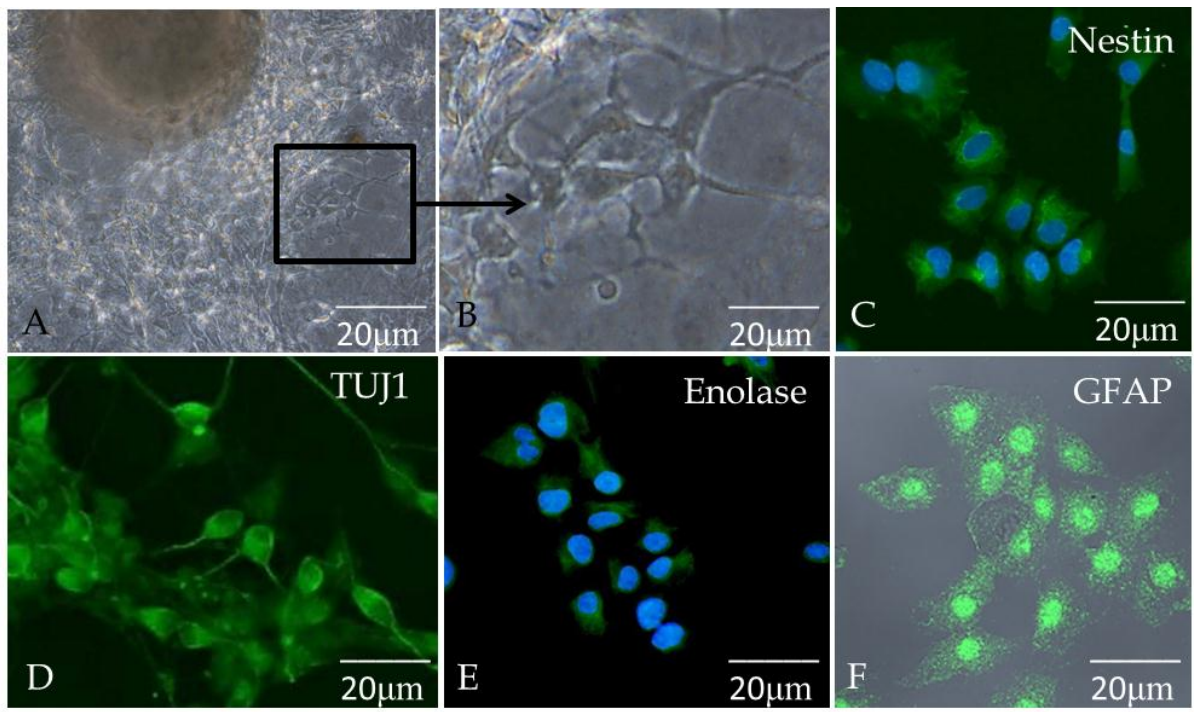

Figure 6. Representative figure of in vitro differentiation of hIDPS-iPSC. A) EBs adherent on Petri dish showing differentiation into neural-like cells after culturing in neurobasal+B27 medium. B) Same as in (A) showing neural-like cells in high magnification. C) Neural-like cells present positive immunostaining with anti-nestin antibody. D) positive immunostaining with anti-TUJ1 antibody. E) Neuron-specific enolase positive immunostaining (green) in hIDPS-iPSC derived neuronal cells. F) Morphological presentation of glial-like cells derived from hIDPS-iPSC. Positive immunostaining for (F) anti-GFAP antibody in glial-like cells. Nucleus stained with DAPI (blue). A, B= Differential interference contrast (DIC). C-E=Epi. F= DIC+Epi. Scale Bars: A-F=20 $\mu \mathrm{m}$.

\subsubsection{Teratoma formation}

To test the pluripotency, iPSC were injected into the testis or intramuscularly into the right and/or left hind leg of severe combined immunodeficient (SCID) mouse. Teratomas formation by SHED/SCAP/DPSCs/DPCs/MStCs-iPSC occurs of nine to eleven weeks after injection and histological examination of the tumor shows representative tissues of three embryonic germ layers, such as gut-like epithelium (endoderm), cartilage (mesoderm), and neuroepithelial rosettes (ectoderm) (Yan et al., 2010; Tamaoki et al., 2010; Oda et al., 2010). Teratomas obtained from IDPSC-iPSC were formed between 5 and 7 weeks after reprogrammed cells injection. The mice injected with parental IDPSC, as expected, did not form teratomas. We observed that teratomas were composed by tissues originated from three primary germ layers. Histological characterization of tumor masses showed that these teratomas includes ectodermal: primitive neural tissues, including neural tube and neural rosettes and retinal epithelium; mesodermal: muscle-like cells and gromerulus-like structures and endodermal tissues: respiratory or gastro-intestinal-like epithelium and glandular-like tissue formation (Fig. 7). Similar to in vitro differentiation, teratomas derived from IDPSC-iPS cells, display strong neuronal commitment forming rosette-, neurosphere- and neural tube-like structures. Neuron-specific enolase, which is a marker of 
neurons and peripheral neuroendocrine tissue, and synaptophysin (a synaptic vesicle glycoprotein), which is found in neuroendocrine cells as well as virtually in all neurons that participate in synaptic transmission in the brain and spinal cord are expressed in neuronal cells produced by IDPSC-iPSC in teratomas (Beltrão-Braga et al., 2011).
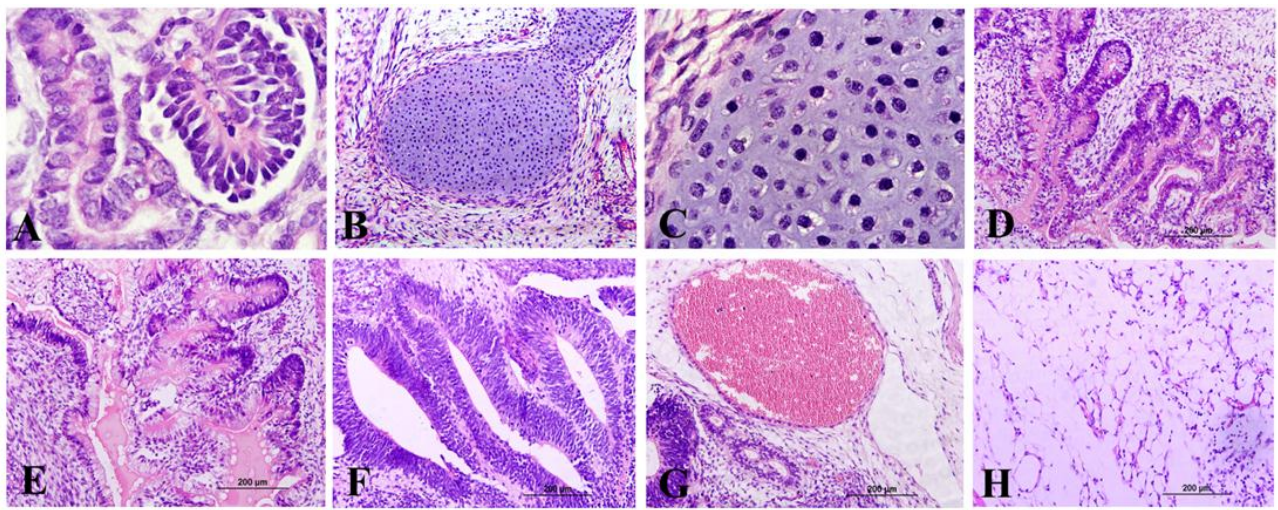

Figure 7. HE stained differentiated tissues from hIDPS-iPSC teratoma seven weeks after transplantation into nude mice right limb. A) Morphogenesis observed during differentiation of hIDPS-iPSC: glomerulus-like structure formation, with Bowman's capsule and convoluted tubule-like structures. B) Cartilage and in (C) Condrocytes-like cells (higher magnification). D) Respiratory-like epithelium. E) Gastrointestinal-like epithelium. F) Neural tubes-like structures. G) Blood vessel. H) Adipose-like tissue. Magnifications: A, C) 100x, B, D-G) 20x, Scale Bar (H) =200 $\mu \mathrm{m}$.

\subsection{HLA typing}

Tamaoki and co-wokers (2010) determined the human leukocyte antigens (HLA) types of 107 dental pulp cells lines in the Japanese population and identified 2 cell lines with homozygous HLA types at all 3 loci (A, B, and DR) examined. They showed that in the Japanese population the frequencies of haplotypes of these 2 homozygous cell lines were estimated to be $8.7 \%$ and $1.5 \%$, data provided by the Japanese Red Cross Society (http://www.bmdc.jrc.or.jp/stat.html). Using these frequencies, the coverage rate for a perfect match of iPSC lines, which were established from these 2 lines was calculated. The authors showed that iPSC lines established from these 2 homozygous cell lines would cover $16.6 \%$ and $3.0 \%$ of the Japanese population, respectively, which corresponds to approximately $20 \%$ of the Japanese population.

\subsection{SHED/SCAP/DPSCs/DPCs/MStCs/IDPSC-iPSC bank and therapeutic use}

One of the major challenge of pluripotent stem cells use in cell therapies is an immunemediated rejection after transplantation. Today, this problem can be overcome by direct reprogramming of patients somatic cells and by creating an iPSC bank consisting of various HLA types thus providing therapeutic tool for the patients, which need cell transplantation free from immune-mediated rejection. Two works reported that the establishment of 50 unique stem cells lines, having homozygous alleles of the 3 HLA loci (A, B, and DR), would cover 
$90 \%$ of the Japanese population with a faultless match of these loci (Nakajima et al., 2007; Nakatsuji et al., 2008). Considering that iPSC derivation is a time consuming process and of elevated cost, it should be necessary for cell therapies and regenerative medicine to establish iPSC banks with a sufficient collection of HLA types, thus avoiding additional costs which are required for iPSC production for each individual patient.

\subsubsection{Requirements of iPSC generation}

In spite of optimistic prognosis in respect of how many iPSC should be produced in order to satisfy their immunological matching within definite human population, several requirements must be challenged before establishing iPSC bank. The principal requirement is a method of reprogramming, which should be safe. Therefore, three major concerns exist in the current reprogramming strategies for clinical applications: (i) the low reprogramming efficiency of human somatic cells makes it difficult to generate patient-specific iPSC, when a small amount of the cells of the patient is used; (ii) carcinogenesis may be caused by genomic integration of retro- or lentiviral fragments into host DNA; and (iii) Myc is an oncogene, which after reactivation might cause malignant tumor formation. Whereas iPSC can be generated by three transcription factors (Oct3/4, Sox2, and Klf4) without Myc, reprogramming efficiency are significantly reduced. Although, several methods of iPSC generation without viral integration have been reported; their efficiencies are extremely low in comparison with viral vectors used for induction of reprogramming (Okita et al., 2008; Stadtfeld et al., 2008; Fusaki et al., 2009; Kaji et al., 2009; Kim et al., 2009; Soldner et al., 2009; Woltjen et al., 2009; Yu et al., 2009; Yusa et al., 2009; Zhou et al., 2009). Next important issue is availability of donor cells, which can provide high efficiency in the generation of non-integrated human iPSC. Therefore, source of the cells also makes its own demand, such as, it should be easily accessible with minimum discomfort for the patient, the procedure of stem cell isolation should be non-invasive, the tissue should be easily processed, the cells should be rapidly proliferating and produced in sufficient quantities, these cells should be young and collected from healthy volunteers. Furthermore, the possibility of genetic abnormalities in donor cells due to ultraviolet (UV) irradiation should be minimized and finally, these cells would be able to be stored in liquid nitrogen for a long time without the loss of their prime characteristics.

Dental pulp stem cells from deciduous and wisdom teeth are an ideal source that meets the majority of aforementioned requirements. The loss of baby (deciduous) teeth occurs naturally and they can be removed with minimal discomfort to the patient during a routine visit to the dentist, in many clinics, as well as wisdom teeth. We also showed that not only cells, but also dental pulp can be cryopreserved and new cells can be obtained later, after thawing (Lizier et al., 2012). Therefore, frozen dental pulp does not require in vitro cultivation in order to produce the cells of donor until he needs these cells for iPSC production and/or clinical treatment. Additionally, several dental pulps from the same individual can be cryopreserved. This elevates a probability of successful MSCs isolation in high quantities. These cells are safer, once they can be used by first and second degree relatives and within all family (Kerkis and Caplan, 2012; Lizier et al., 2012). 


\subsubsection{Perspectives of iPSC therapeutic use}

Currently, iPSC are used to understand human diseases, including Alzheimer's disease, Parkinson's disease, cardiovascular disease, diabetes, and amyotrophic lateral sclerosis (ALS), to develop and screen bioactive molecules - candidate to therapeutic drugs and to identify molecules or genes implicated in tissue regeneration. These in vitro studies enable researchers to understand fundamental principles of iPSC function and differentiation, which further will provide knowledge, necessary for therapeutic use of iPSC. Based on this knowledge multiple pre-clinical and clinical protocols will be produced optimizing iPSC transplantation in diverse animal and human diseases thus becoming a tool in cellreplacement therapy. Therefore, iPSC in the future may have tremendous clinical potential when highly efficient and safe protocols of generation of reprogrammed stem cells will be developed. Increasing our understanding of the molecular mechanisms that underlie reprogramming, we will be able to identify the cell types and methods of reprogramming, which will minimize DNA alterations, and conditions of iPSC cultivation that will allow widespread use of these cells in clinic. The scientists, however, should answer the question if iPSC are truly equivalent to human ES cells. Although iPSC potential for regenerative medicine is great, our current knowledge about iPSC variability, and utility must also increase greatly before iPSC became a standard tool for regenerative medicine.

\section{Final considerations}

Different dental tissues, which include apical papilla, primary exfoliated deciduous and permanent teeth, as well as wisdom teeth were used to derivate iPSC. The data obtained by different authors indicate that these tissues can be easily isolated and MSCs cells in sufficient quantities can be obtained. MSCs in vivo are reversibly arrested cells, which are localized in their niches maintaining their temporarily quiescent state. They differ from terminally differentiated cells by developmental path that involves a set of increasingly committed stages of specialization. The fact that these cells are undifferentiated cells suggests that their reprogramming will occur more easily than that of terminally differentiated cells. Indeed, all studies demonstrated that in SHED/SCAP/DPSCs/DPCs/MStCs/IDPSC the reprogramming process occurs more easily than in human fibroblasts used as a control, under the similar protocols and vectors used for transduction, which showed to be efficient. It was also possible to reprogram MSCs from wisdom teeth using only three factors (without c-Myc) avoiding future implications with potential risks of oncogene use (Yu et al., 2007). For the clinical applications of stem cells xenogeneic reagents pose the risk of a severe immune response, and the transmission of viral or bacterial infections, prions, and unidentified zoonosis. We demonstrated that isolation of IDPSC-iPSC can occur under feeder-free conditions on matrigel-coated dishes. Clinical stem cell therapy trials are ongoing, which request a strong focus on the safety and quality of in vitro expanded stem cell transplants. By replacing xenogeneic products with a defined xeno-free medium, the safety and quality of the cells with therapeutic potential may be enhanced significantly. Similar to human ES cells, dental MSCs derived iPSC form compact colony and retain immortal growth characteristics in culture. They express markers charac- 
teristic of pluripotency including Nanog, Oct-4, SSEA-3, SSEA-4, TRA-1-60, TRA-1-81, exhibit high telomerase activity and have stable karyotype. Controversies were observed in methylation status of $\mathrm{CpG}$ in the promoter regions of Nanog and Oct4 in dental tissue derived iPSC. Thus iPSC-derived from MStCs from wisdom teeth have their promoters of Oct4 and Nanog highly unmethylated after reprogramming (Yan et al., 2010; Oda et al., 2010). The SHED-/ DPSC-iPSC had less methylated sites of Oct4 promoter than the non-transduced cells (Yan et al., 2010). What ensues at the molecular level during the reprogramming process, however, is not fully understood and is the current focus in iPSC research (Amabile and Meissner, 2009). Furthermore, dental tissue derived iPSC exhibits differentiation potential like human ES cells and can differentiate in vitro and in vivo into cells of all three primary germ layers (Takahashi et al., 2007; Yu et al., 2007).

In cell types with endogenously expression of one or more of the factors that induce pluripotency, such as neural cells that strongly express Sox2, pluripotency may be induced more easily or even with only a subset of factors (de Souza, 2010). In accordance, we observed that in hIDPSC, which express these factors, but at low level, the reprogramming was speedier, when compared with other dental tissue derived stem cells. During reprogramming, the integration the 4 factors into the genome of the transduced SHED/SCAP/DPSC-iPSC occurred (Yan et al., 2010). Currently, the nonintegrating reprogramming approaches, which include adenoviruses, plasmid- and episomal vector-based methods, and delivery of reprogramming factors directly as proteins have been developed. Additionally, other factors have been identified that can substitute the four Yamanaka's traditional transcription factors. Thus, Klf227 and Klf5 can replace Klf4, Sox1 and Sox3 can replace Sox2, and n-Myc and I-Myc can replace c-Myc (Nakagawa et al., 2008). Nr5a2 (Nuclear receptor subfamily 5, group A, member 2) can be used to substitute Oct- 4 in the reprogramming of murine somatic cells (Heng et al., 2010). Some small molecules as the histone deacetylase inhibitor valproic acid can replace Klf4 and c-Myc for reprogramming human fibroblasts (Huangfu et al., 2008; Lin et al., 2009).

The creation of patient-specific stem cell lines is relevant for the study of basic biology, molecular mechanisms of various diseases, for drug discovery and for treating a number of human degenerative diseases without evoking immune rejection. HLA typing of DPC lines (Tamaoki et al., 2010) is of extreme importance because allows to limit the number of human iPSC, which should be obtained for each definite human population, thus avoiding unnecessary elevated costs of iPSC for cell therapies and regenerative medicine. So far, human iPSC have been used for the study of the reprogramming process itself and establishment of disease-specific cell lines and the differentiation of these cell lines into the different cell types affected by the disease, such as, spinal motor neurons, dopaminergic neurons and cardiomyocytes derived from patients suffering from amyotrophic lateral sclerosis (Dimos et al., 2008), spinal muscular atrophy (Ebert et al., 2009), sporadic Parkinson's disease (Soldner et al., 2009). Exploration of iPSC is still in its infancy, and understanding the true potential of these cells requires continued research, comprehension and profound comparisons with human ES cells. 


\section{Acknowledgements}

The authors thank Dr. Alysson Muotri from University of California San Diego, Dr. Patricia Beltrao-Braga and Dr. Paulo Maiorka from University of Sao Paulo for their contribution in our research.

\section{Author details}

Nelson F. Lizier, Irina Kerkis and Cristiane V. Wenceslau

Laboratory of Genetics, Butantan Institute and Department of Morphology and Genetics, Federal University of Sao Paulo, Brazil

\section{References}

[1] Aasen T, Raya A, Barrero MJ, Garreta E, Consiglio A, Gonzalez F, Vassena R, Bilić J, Pekarik V, Tiscornia G, Edel M, Boué S, Izpisúa Belmonte JC. (2008) Efficient and rapid generation of induced pluripotent stem cells from human keratinocytes. Nat Biotechnol. Nov; 26(11):1276-84. Epub 2008 Oct 17.

[2] Amabile G, Meissner A. (2009) Induced pluripotent stem cells: current progress and potential for regenerative medicine. Trends Mol Med. Feb; 15(2):59-68. Epub 2009 Jan 21.

[3] Banito A, Rashid ST, Acosta JC, Li S, Pereira CF, Geti I, Pinho S, Silva JC, Azuara V, Walsh M, Vallier L, Gil J. (2009) Senescence impairs successful reprogramming to pluripotent stem cells. Genes Dev. Sep 15; 23(18):2134-9. Epub 2009 Aug 20.

[4] Beltrão-Braga PC, Pignatari GC, Maiorka PC, Oliveira NA, Lizier NF, Wenceslau CV, Miglino MA, Muotri AR, Kerkis I. (2011) Feeder-free derivation of induced pluripotent stem cells from human immature dental pulp stem cells. Cell Transplant.

[5] Bilic J, Izpisua Belmonte JC. (2012) Concise review: Induced pluripotent stem cells versus embryonic stem cells: close enough or yet too far apart? Stem Cells. Jan; 30(1): 33-41.

[6] Caplan AI. (1991) Mesenchymal stem cells. J Orthop Res. Sep; 9(5):641-50.

[7] Cho YW, Hong T, Hong S, Guo H, Yu H, Kim D, Guszczynski T, Dressler GR, Copeland TD, Kalkum M, Ge K. (2007) PTIP associates with MLL3- and MLL4-containing histone H3 lysine 4 methyltransferase complex. J Biol Chem. Jul 13; 282(28): 20395-406. Epub 2007 May 11. 
[8] Conkright, M. D., Wani, M. A., Anderson, K. P., and Lingrel, J. B. (1999) A gene encoding an intestinal-enriched member of the Krüppel-like factor family expressed in intestinal epithelial cells. Nucleic Acids Res. 27, 1263-1270

[9] de Souza N. (2010) Primer: induced pluripotency. Nat Methods. Jan; 7(1):20-1.

[10] Dimos JT, Rodolfa KT, Niakan KK, Weisenthal LM, Mitsumoto H, Chung W, Croft GF, Saphier G, Leibel R, Goland R, Wichterle H, Henderson CE, Eggan K. (2008) Induced pluripotent stem cells generated from patients with ALS can be differentiated into motor neurons. Science. Aug 29; 321(5893):1218-21.

[11] Dupin E, Calloni G, Real C, Gonçalves-Trentin A, Le Douarin NM. (2007) Neural crest progenitors and stem cells. C R Biol. Jun-Jul; 330(6-7):521-9.

[12] Ebert AD, Yu J, Rose FF Jr, Mattis VB, Lorson CL, Thomson JA, Svendsen CN. (2009) Induced pluripotent stem cells from a spinal muscular atrophy patient. Nature. Jan 15; 457(7227):277-80. Epub 2008 Dec 21.

[13] Friedenstein AJ, Gorskaja JF, Kulagina NN. (1976) Fibroblast precursors in normal and irradiated mouse hematopoietic organs. Exp Hematol. Sep; 4(5):267-74.

[14] Fusaki N, Ban H, Nishiyama A, Saeki K, Hasegawa M. (2009) Efficient induction of transgene-free human pluripotent stem cells using a vector based on Sendai virus, an RNA virus that does not integrate into the host genome. Proc Jpn Acad 85: 348-362.

[15] Giordano G, La Monaca G, Annibali S, Cicconetti A, Ottolenghi L. (2011) Stem cells from oral niches: a review. Ann Stomatol (Roma). Jan; 2(1-2):3-8. Epub 2011 Jul 18.

[16] Heng JC, Feng B, Han J, Jiang J, Kraus P, Ng JH, Orlov YL, Huss M, Yang L, Lufkin T, Lim B, Ng HH. (2010) The nuclear receptor Nr5a2 can replace Oct4 in the reprogramming of murine somatic cells to pluripotent cells. Cell Stem Cell. Feb 5; 6(2): 167-74. Epub 2010 Jan 21.

[17] Huangfu D, Osafune K, Maehr R, Guo W, Eijkelenboom A, Chen S, Muhlestein W, Melton DA. (2008) Induction of pluripotent stem cells from primary human fibroblasts with only Oct4 and Sox2. Nat Biotechnol. Nov; 26(11):1269-75.

[18] Ikeda E, Yagi K, Kojima M, Yagyuu T, Ohshima A, Sobajima S, Tadokoro M, Katsube Y, Isoda K, Kondoh M, Kawase M, Go MJ, Adachi H, Yokota Y, Kirita T, Ohgushi H. (2008) Multipotent cells from the human third molar: feasibility of cell-based therapy for liver disease. Differentiation. May; 76(5):495-505.

[19] Kaji K, Norrby K, Paca A, Mileikovsky M, Mohseni P, Woltjen K. (2009) Virus-free induction of pluripotency and subsequent excision of reprogramming factors. Nature 458: 771-775.

[20] Kerkis I, Caplan AI. (2012) Stem cells in dental pulp of deciduous teeth. Tissue Eng Part B Rev. Apr;18(2):129-38

[21] Kerkis I, Kerkis A, Dozortsev D, Stukart-Parsons GC, Gomes Massironi SM, Pereira LV, Caplan AI, Cerruti HF (2006)Isolation and characterization of a population of im- 
mature dental pulp stem cells expressing OCT-4 and other embryonic stem cell markers. Cells Tissues Organs; 184(3-4):105-16.

[22] Kim D, Kim CH, Moon JI, Chung YG, Chang MY, Han BS, Ko S, Yang E, Cha KY, Lanza R, et al. (2009) Generation of human induced pluripotent stem cells by direct delivery of reprogramming proteins. Cell Stem Cell 4: 472-476.

[23] Le Douarin NM, Brito JM, Creuzet S (2007) Role of the neural crest in face and brain development. Brain Res Rev. Oct; 55(2):237-47.

[24] Le Douarin NM, Calloni GW, Dupin E (2008) The stem cells of the neural crest. Cell Cycle 7: 1013-9.

[25] Le Douarin NM, Creuzet S, Dupin E (2004) Neural crest cell plasticity and its limits. Development 131: 4637-50

[26] Le Douarin NM, Dupin E (2003) Multipotentiality of the neural crest. Curr Opin Genet Dev 13: 529-36.

[27] Le Douarin NM, Dupin E. (2012)The neural crest in vertebrate evolution. Curr Opin Genet Dev. Jul 6. [Epub ahead of print]

[28] Lewitzky M, Yamanaka S. (2007) Reprogramming somatic cells towards pluripotency by defined factors. Curr Opin Biotechnol. Oct; 18(5):467-73.

[29] Lin T, Ambasudhan R, Yuan X, Li W, Hilcove S, Abujarour R, Lin X, Hahm HS, Hao E, Hayek A, et al. (2009) A chemical platform for improved induction of human iPSCs. Nat Methods 6: 805-808.

[30] Liu SV (2008) iPS cells: a more critical review. Stem Cells Dev. Jun;17(3):391-7.

[31] Lizier NF, Kerkis A, Gomes CM, Hebling J, Oliveira CF, Caplan AI, Kerkis I (2012) Scaling-up of dental pulp stem cells isolated from multiple niches. PLoS One; 7(6):e39885. Epub 2012 Jun 29.

[32] Maherali N, Hochedlinger K (2008) Induced pluripotency of mouse and human somatic cells. Cold Spring Harb Symp Quant Biol. 8; 73:157-62.

[33] Marchetto MC, Yeo GW, Kainohana O, Marsala M, Gage FH, Muotri AR. (2009) Transcriptional signature and memory retention of human-induced pluripotent stem cells. PLoS One. Sep 18; 4(9):e7076.

[34] McDevitt TC, Palecek SP (2008) Innovation in the culture and derivation of pluripotent human stem cells. Curr Opin Biotechnol. Oct; 19(5):527-33

[35] Miura, M., Gronthos, S., Zhao, M., Lu, B., Fisher, L.W., et al. (2003) SHED: Stem cells from human exfoliated deciduous teeth. Proc Natl Acad Sci U S A 100, 5807.

[36] Muchkaeva IA, Dashinimaev EB, Terskikh VV, Sukhanov YV, Vasiliev AV (2012) Molecular mechanisms of induced pluripotency. Acta Naturae. Jan; 4(1):12-22. 
[37] Murata K, Kouzarides T, Bannister AJ, Gurdon JB (2010) Histone H3 lysine 4 methylation is associated with the transcriptional reprogramming efficiency of somatic nuclei by oocytes. Epigenetics Chromatin. Feb 4; 3(1):4.

[38] Nakagawa M, Koyanagi M, Tanabe K, Takahashi K, Ichisaka T, Aoi T, Okita K, Mochiduki Y, Takizawa N, Yamanaka S (2008) Generation of induced pluripotent stem cells without Myc from mouse and human fibroblasts. Nat Biotechnol. Jan; 26(1): 101-6. Epub 2007 Nov 30.

[39] Nakajima F, Tokunaga K, Nakatsuji N (2007) Human leukocyte antigen matching estimations in a hypothetical bank of human embryonic stem cell lines in the Japanese population for use in cell transplantation therapy. Stem Cells. Apr; 25(4):983-5. Epub 2006 Dec 21.

[40] Nakatsuji N, Nakajima F, Tokunaga K (2008) HLA-haplotype banking and iPS cells. Nat Biotechnol. Jul; 26(7):739-40.

[41] Nishikawa S, Goldstein RA, Nierras CR (2008) The promise of human induced pluripotent stem cells for research and therapy. Nat Rev Mol Cell Biol. Sep; 9(9):725-9.

[42] Oda Y, Yoshimura Y, Ohnishi H, Tadokoro M, Katsube Y, Sasao M, Kubo Y, Hattori K, Saito S, Horimoto K, Yuba S, Ohgushi H (2010) Induction of pluripotent stem cells from human third molar mesenchymal stromal cells. J Biol Chem. Sep 17; 285(38): 29270-8.

[43] Ohnuki M, Takahashi K, Yamanaka S (2009) Generation and characterization of human induced pluripotent stem cells. Curr Protoc Stem Cell Biol. Jun; Chapter 4: Unit 4A.2.

[44] Okita K, Nakagawa M, Hyenjong H, Ichisaka T, Yamanaka S (2008) Generation of mouse induced pluripotent stem cells without viral vectors. Science. Nov 7; 322(5903):949-53. Epub 2008 Oct 9.

[45] Ooi L, Sidhu K, Poljak A, Sutherland G, O'Connor MD, Sachdev P, Münch G (2012) Induced pluripotent stem cells as tools for disease modelling and drug discovery in Alzheimer's disease. J Neural Transm. Jun 13.

[46] Patel SR, Kim D, Levitan I, Dressler GR (2007) The BRCT-domain containing protein PTIP links PAX2 to a histone H3, lysine 4 methyltransferase complex. Dev Cell. Oct; 13(4):580-92.

[47] Shields, J. M., Christy, R. J., and Yang, V. W. (1996) Identification and characterization of a gene encoding a gut-enriched Krüppel-like factor expressed during growth arrest. J. Biol. Chem. 271, 20009-20017.

[48] Soldner F, Hockemeyer D, Beard C, Gao Q, Bell GW, Cook EG, Hargus G, Blak A, Cooper O, Mitalipova M, Isacson O, Jaenisch R (2009) Parkinson's disease patient-derived induced pluripotent stem cells free of viral reprogramming factors. Cell. Mar 6; 136(5):964-77. 
[49] Stadtfeld M, Nagaya M, Utikal J, Weir G, Hochedlinger K (2008) Induced pluripotent stem cells generated without viral integration. Science 322: 945-949.

[50] Takahashi K, Tanabe K, Ohnuki M, Narita M, Ichisaka T, Tomoda K, Yamanaka S. (2007) Induction of pluripotent stem cells from adult human fibroblasts by defined factors. Cell 131: 861-872.

[51] Takahashi K, Yamanaka S (2006) Induction of pluripotent stem cells from mouse embryonic and adult fibroblast cultures by defined factors. Cell. 126(4): 663-76.

[52] Takeda T, Tezuka Y, Horiuchi M, Hosono K, Iida K, Hatakeyama D, Miyaki S, Kunisada T, Shibata T, Tezuka K. (2008) Characterization of dental pulp stem cells of human tooth germs. J Dent Res. Jul; 87(7):676-81.

[53] Tamaoki N, Takahashi K, Tanaka T, Ichisaka T, Aoki H, Takeda-Kawaguchi T, Iida K, Kunisada T, Shibata T, Yamanaka S, Tezuka K (2010) Dental pulp cells for induced pluripotent stem cell banking. J Dent Res. Aug; 89(8):773-8.

[54] van Attikum H, Gasser SM (2009) Crosstalk between histone modifications during the DNA damage response. Trends Cell Biol. May; 19(5):207-17. Review.

[55] Woltjen K, Michael IP, Mohseni P, Desai R, Mileikovsky M, Hamalainen R, Cowling R, Wang W, Liu P, Gertsenstein M, Stadtfeld and Hochedlinger et al. (2009) piggyBac transposition reprograms fibroblasts to induced pluripotent stem cells. Nature 458: 766-770

[56] Yan, X., Qin, H., Qu, C., Tuan, R.S., Shi, S., and Huang, G.T (2010) iPS cell reprogrammed from human mesenchymal-like stem/progenitor cells of dental tissue origin. Stem Cells Dev 19, 469.

[57] Yu J, Hu K, Smuga-Otto K, Tian S, Stewart R, Slukvin II, Thomson JA (2009) Human induced pluripotent stem cells free of vector and transgene sequences. Science 324: 797801.

[58] Yu J, Thomson JA. (2008) Pluripotent stem cell lines. Genes Dev.; 22(15):1987-97.

[59] Yu J, Vodyanik MA, Smuga-Otto K, Antosiewicz-Bourget J, Frane JL, Tian S, Nie J, Jonsdottir GA, Ruotti V, Stewart R, et al. (2007) Induced pluripotent stem cell lines derived from human somatic cells. Science 318: 1917-1920.

[60] Yusa K, Rad R, Takeda J, Bradley A (2009) Generation of transgene-free induced pluripotent mouse stem cells by the piggyBac transposon. Nat Methods 6: 363-369.

[61] Zhao R, Daley GQ (2008) From fibroblasts to iPS cells: induced pluripotency by defined factors. J Cell Biochem.; 105(4): 949-55

[62] Zhou W, Freed CR (2009) Adenoviral gene delivery can reprogram human fibroblasts to induced pluripotent stem cells. Stem Cells 27: 2667-2674.

[63] Zouboulis CC, Adjaye J, Akamatsu H, Moe-Behrens G, Niemann C (2008) Human skin stem cells and the ageing process. Exp Gerontol. Nov; 43(11):986-97. 
